\title{
JUSTIFICATION OF THE EXISTENCE OF A GROUP OF ASYMPTOTICS OF THE GENERAL FIFTH PAINLEVÉ TRANSCENDENT
}

\author{
YOUMIN LU and ZHOUDE SHAO
}

Received 10 February 2004

\begin{abstract}
There are several existing ways in developing the asymptotics of the Painlevé transcendents. But it is always a hard task to justify the existence of these asymptotics. In this note, we apply the successive approximation to the general fifth Painlevé equation and rigorously prove the existence of a group of asymptotics of its solutions.
\end{abstract}

2000 Mathematics Subject Classification: 34E99.

1. Introduction. The mathematical and physical significance of the six Painlevé transcendents has been well established. Their mathematical importance originates from the work by Painlevé [7, 8] and Garnier [2]. Their physical significance follows their applicability to a wide range of important physical problems, such as nonlinear waves in quantum field theory and statistical mechanics [6]. There have been many results on the asymptotics of the Painlevé transcendents. In 1980, Hastings and McLeod [4] developed a method and applied it to the second Painlevé equation. In their paper, they rigorously proved the existence of a group of asymptotics to the second Painlevé equation and obtained a connection formula of the asymptotics. In 1997, Abdullayev [1] further developed the ideas used by Hastings and McLeod, "linearized" a special case of the fourth Painlevé equation and proved the existence of a group of its asymptotics. In [5], we studied the general fifth Painlevé equation

$(\mathrm{PV})$

$$
y^{\prime \prime}=\left(\frac{1}{2 y}+\frac{1}{y-1}\right) y^{\prime 2}-\frac{y^{\prime}}{x}+\frac{(y-1)^{2}}{x^{2}}\left(\alpha y+\frac{\beta}{y}\right)+\frac{\gamma y}{x}-\frac{\delta y(y+1)}{y-1}
$$

and developed several groups of asymptotics of its negative solutions. In this note, we apply Abdullayev's idea to (PV) and prove the following theorem.

THEOREM 1.1. If $\delta>0$, there exists a two-parametric family $y(x)=y\left(x, a, \phi_{0}\right)$ of solutions of $(P V)$ such that the following asymptotics hold as $x \rightarrow \infty$ :

$$
\begin{gathered}
y(x)=-1-4 a x^{-1 / 2} \cos \phi+O\left(x^{-1}\right), \\
y^{\prime}(x)=4 a x^{-1 / 2} \sin \phi+O\left(x^{-1}\right), \\
\phi=x-a^{2} \ln x+\phi_{0} .
\end{gathered}
$$


It is important to notice that this theorem does not only show the existence of the solutions, but also shows the differentiability of the asymptotics.

Because we are studying (PV)s with the parameter $\delta>0$, and $\delta$ can be scaled to any positive number when it is positive, we simply prove the theorem for $\delta=2$. In order to "linearize" (PV), we first use the transformation

$$
y(x)=-\tan ^{2} v(x)
$$

to transform (PV) into the equation

$$
v^{\prime \prime}+x^{-1} v^{\prime}=\frac{x^{-2}}{2}\left(\alpha \tan v \sec ^{2} v+\beta \cot v \csc ^{2} v\right)+\frac{\gamma}{4} x^{-1} \sin 2 v+\frac{1}{4} \sin 4 v
$$

It is clear that transformation (1.3) maps the region $0 \leq v \leq \pi / 2$ onto the region $-\infty \leq$ $y \leq 0 . y=0$ and $y=-\infty$ are corresponding to $v=0$ and $v=\pi / 2$, respectively.

Corresponding to the asymptotics of $y(x)$ in the theorem, we now seek formally a solution of (1.4) in the form

$$
v(x)=\frac{\pi}{4}+a x^{-1 / 2} \cos \phi+A x^{-1} .
$$

Substituting (1.5) into (1.4), we find $A=\gamma / 4$.

2. Proof of Theorem 1.1. First, we set

$$
v(x)=\frac{\pi}{4}+x^{-1 / 2} W(x),
$$

and "linearize" each term in the right-hand side of (1.4). Using the power series of the trigonometric functions, we get

$$
\begin{gathered}
\tan \left(\frac{\pi}{4}+x^{-1 / 2} W\right) \sec ^{2}\left(\frac{\pi}{4}+x^{-1 / 2} W\right)=2+x^{-1 / 2} W+20 x^{-1} W^{2}+x^{-3 / 2} f_{1}(W), \\
\cot \left(\frac{\pi}{4}+x^{-1 / 2} W\right) \csc ^{2}\left(\frac{\pi}{4}+x^{-1 / 2} W\right)=2-x^{-1 / 2} W+20 x^{-1} W^{2}+x^{-3 / 2} f_{2}(W), \\
\sin \left(\frac{\pi}{2}+2 x^{-1 / 2} W\right)=1-2 x^{-1} W^{2}+x^{-2} f_{3}(W) \\
\sin \left(\pi+4 x^{-1 / 2} W\right)=-4 x^{-1 / 2} W+\frac{32}{3} x^{-3 / 2} W^{3}+x^{-5 / 2} f_{4}(W)
\end{gathered}
$$

where $f_{1}(W)=O\left(W^{3}\right), f_{2}(W)=O\left(W^{3}\right), f_{3}(W)=O\left(W^{4}\right)$, and $f_{4}(W)=O\left(W^{5}\right)$. Substituting (2.1) and (2.2) into (1.4), we can get a "linearized" form of the equation

$$
\begin{aligned}
W^{\prime \prime}(x)+W(x)= & \frac{\gamma}{4} x^{-1 / 2}+\frac{8}{3} x^{-1} W^{3}-\frac{\gamma}{2} x^{-3 / 2} W^{2} \\
& +(\alpha+\beta) x^{-3 / 2}-\frac{1}{4} x^{-2} W+x^{-2} f_{5}(W),
\end{aligned}
$$

where $f_{5}(W)=(\alpha / 2) x^{-1} f_{1}(W)+(\beta / 2) x^{-1} f_{2}(W)+(\gamma / 4) x^{-1 / 2} f_{3}(W)+(1 / 4) f_{4}(W)$ and $d f_{5} / d W$ is bounded when $\left|x^{-1 / 2} W\right| \leq c_{0}<\pi / 4$. 
To further "linearize" the equation, we need the following transformation:

$$
W(x)=a \cos \phi+\frac{\gamma}{4} x^{-1 / 2}+W_{1}(x) .
$$

Substituting this transformation into (2.3), we obtain the equation

$$
\begin{aligned}
W_{1}^{\prime \prime}(x)+f(x) W_{1}(x)= & g_{0}(x)+x^{-3 / 2} g_{1}(x) W_{1}+x^{-1} g_{2}(x) W_{1}^{2} \\
& +x^{-1} g_{3}(x) W_{1}^{3}+x^{-2} f_{5}(W),
\end{aligned}
$$

where

$$
\begin{gathered}
f(x)=1-4 a^{2} x^{-1}-4 a^{2} \cos 2 \phi, \\
g_{0}(x)=\frac{2}{3} a^{3} x^{-1} \cos 3 i-\frac{a^{2} \gamma}{2} x^{-3 / 2} \cos ^{2} \phi+(\alpha+\gamma) x^{-3 / 2}+2 a^{2} \gamma x^{-2} \cos ^{2} \phi \\
-\frac{a}{4} x^{-2} \cos \phi-\frac{a \gamma^{2}}{4} x^{-5 / 2} \cos \phi+\frac{a \gamma^{2}}{2} x^{-3} \cos \phi+a^{5} x^{-2} \cos \phi \\
+a^{3} x^{-2} \sin \phi-\frac{3 \gamma}{16} x^{-5 / 2}-\frac{\gamma^{3}}{32} x^{-3 / 2}, \\
g_{1}(x)=-a \gamma+4 a \gamma x^{-1 / 2} \cos \phi-\frac{1}{4} x^{-1 / 2}-\frac{\gamma^{2}}{4} x^{-1}+\frac{\gamma^{2}}{2} x^{-3 / 2}, \\
g_{2}(x)=8 a \cos \phi-\frac{\gamma}{2} x^{-1 / 2}+2 \gamma x^{-1}, \\
g_{3}(x)=\frac{8}{3} .
\end{gathered}
$$

To apply the successive approximation to (2.5), we first need to solve the equation

$$
w^{\prime \prime}(x)+f(x) w(x)=0
$$

where $f(x)$ is the function defined above.

LEMMA 2.1. Equation (2.7) has a fundamental system of solutions with the following asymptotics representations:

$$
\begin{aligned}
w_{1}(x)= & \left(\cos \left(x-2 a^{2} \ln x\right)+\sin \left(x-2 a^{2} \ln x\right)\right) \\
& \times e^{(1 / 2)\left(\cos 2\left(a^{2} \ln x+\phi_{0}\right)+\sin 2\left(a^{2} \ln x+\phi_{0}\right)\right)}+O\left(x^{-1}\right), \\
w_{2}(x)= & \left(\cos \left(x-2 a^{2} \ln x\right)-\sin \left(x-2 a^{2} \ln x\right)\right) \\
& \times e^{(1 / 2)\left(\cos 2\left(a^{2} \ln x+\phi_{0}\right)-\sin 2\left(a^{2} \ln x+\phi_{0}\right)\right)}+O\left(x^{-1}\right), \\
w_{1}^{\prime}(x)= & \left(\cos \left(x-2 a^{2} \ln x\right)-\sin \left(x-2 a^{2} \ln x\right)\right) \\
& \times e^{(1 / 2)\left(\cos 2\left(a^{2} \ln x+\phi_{0}\right)+\sin 2\left(a^{2} \ln x+\phi_{0}\right)\right)}+O\left(x^{-1}\right), \\
w_{2}^{\prime}(x)= & \left(-\cos \left(x-2 a^{2} \ln x\right)-\sin \left(x-2 a^{2} \ln x\right)\right) \\
& \times e^{(1 / 2)\left(\cos 2\left(a^{2} \ln x+\phi_{0}\right)-\sin 2\left(a^{2} \ln x+\phi_{0}\right)\right)}+O\left(x^{-1}\right) .
\end{aligned}
$$


Proof. Harris Jr. and Lutz [3] and Abdullayev [1] have proved some results on similar equations. We use their ideas in this proof. First, we use the transformation

$$
\vec{w}=\left(\begin{array}{cc}
\cos \psi & \sin \psi \\
-\psi^{\prime} \sin \psi & \psi^{\prime} \cos \psi
\end{array}\right) \vec{u}
$$

where $\vec{w}=\left(\begin{array}{c}w \\ w^{\prime}\end{array}\right), \vec{u}=\left(\begin{array}{l}u_{1} \\ u_{2}\end{array}\right)$, and $\psi=x-2 a^{2} \ln x$, to transform (2.7) into the following system of linear equations:

$$
\begin{aligned}
& \vec{u}^{\prime}=\left[a^{2} x^{-1}\left(\begin{array}{cc}
-\sin 2(\phi-\psi) & \cos 2(\phi-\psi) \\
\cos 2(\phi-\psi) & -\sin 2(\phi-\psi)
\end{array}\right)\right. \\
& \left.+a^{2} x^{-1}\left(\begin{array}{cc}
\sin 2(\phi+\psi) & \cos 2(\phi+\psi)-2 \cos 2 \phi \\
\cos 2(\phi+\psi)+2 \cos 2 \phi & \sin 2(\phi+\psi)
\end{array}\right)+R_{1}(x)\right] \vec{u},
\end{aligned}
$$

where $R_{1}(x)=\left(\begin{array}{ll}r_{11}(x) & r_{12}(x) \\ r_{13}(x) & r_{14}(x)\end{array}\right)$ with $r_{1 j}(x)=O\left(x^{-2}\right)$ for $j=1,2,3,4$.

The first matrix in the right-hand side clearly causes some problems because $\phi-\psi=$ $a^{2} \ln x+\phi_{0}$. We solve this problem by diagonalizing this matrix first. Let

$$
\vec{u}=\left(\begin{array}{cc}
1 & 1 \\
1 & -1
\end{array}\right) \vec{z}
$$

Then,

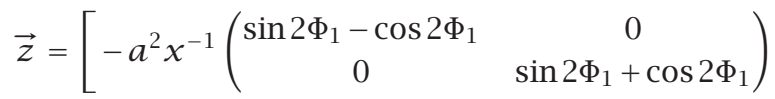

$$
\begin{aligned}
& \left.+a^{2} x^{-1}\left(\begin{array}{cc}
\sin 2 \Psi_{1}+\cos 2 \Psi_{1} & 2 \cos 2 \phi \\
-2 \cos 2 \phi & \sin 2 \Psi_{1}-\cos 2 \Psi_{1}
\end{array}\right)+R_{2}(x)\right] \vec{z},
\end{aligned}
$$

where $\Phi_{1}=\phi-\psi, \Psi_{1}=\phi+\psi$, and $R_{2}(x)=\left(\begin{array}{ll}r_{21}(x) & r_{22}(x) \\ r_{23}(x) & r_{24}(x)\end{array}\right)$ with $r_{2 j}(x)=O\left(x^{-2}\right)$ for $j=1,2,3,4$. To deal with the second matrix whose elements are only conditionally integrable up to infinity, we apply the transformation

$$
\vec{z}=\left(\begin{array}{cc}
1 & a^{2} x^{-1} \sin 2 \phi \\
-a^{2} x^{-1} \sin 2 \phi & 1
\end{array}\right) \vec{h}
$$

where $a^{2} x^{-1} \sin 2 \phi$ is the major term of the integral of $2 a^{2} x^{-1} \cos 2 \phi$, to (2.12) and obtain

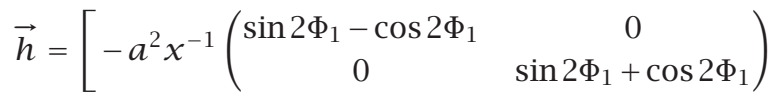

$$
\begin{aligned}
& \left.+a^{2} x^{-1}\left(\begin{array}{cc}
\sin 2 \Psi_{1}+\cos 2 \Psi_{1} & 0 \\
0 & \sin 2 \Psi_{1}-\cos 2 \Psi_{1}
\end{array}\right)+R_{3}(x)\right] \vec{h},
\end{aligned}
$$


where $R_{3}(x)=\left(\begin{array}{l}r_{31}(x) \\ r_{33}(x) \\ r_{34}(x)\end{array}\right)$ with $r_{3 j}(x)=O\left(x^{-2}\right)$ for $j=1,2,3,4$. We solve the diagonalized part of (2.14) and obtain

$$
\begin{aligned}
h_{10} & =\exp \left\{a^{2} \int_{x_{0}}^{x} t^{-1}\left(\cos 2 \Phi_{1}-\sin 2 \Phi_{1}+\sin 2 \Psi_{1}+\cos 2 \Psi_{1}\right) d t\right\} \\
& =e^{(1 / 2)\left(\cos 2\left(a^{2} \ln x+\phi_{0}\right)+\sin 2\left(a^{2} \ln x+\phi_{0}\right)\right)}+O\left(x^{-1}\right), \\
h_{20} & =\exp \left\{a^{2} \int_{x_{0}}^{x} t^{-1}\left(-\cos 2 \Phi_{1}-\sin 2 \Phi_{1}+\sin 2 \Psi_{1}-\cos 2 \Psi_{1}\right) d t\right\} \\
& =e^{(1 / 2)\left(\cos 2\left(a^{2} \ln x+\phi_{0}\right)-\sin 2\left(a^{2} \ln x+\phi_{0}\right)\right)}+O\left(x^{-1}\right) .
\end{aligned}
$$

Now, we can let

$$
\vec{h}=h_{0} \vec{c}
$$

where $h_{0}=\operatorname{diag}\left(h_{10}, h_{20}\right)$ and $\vec{c}=\left(\begin{array}{l}c_{1} \\ c_{2}\end{array}\right)$, and convert (2.14) to an integral equation

$$
\vec{c}=\vec{c}_{0}+\int_{\infty}^{x} h_{0}^{-1} R_{3}(t) h_{0} \vec{c} d t
$$

It is clear now that (2.17) has solutions $\vec{c}=\vec{c}_{0}+O\left(x^{-1}\right)$. Thus, we obtain a solution matrix

$$
\left(\begin{array}{cc}
\cos \psi & \sin \psi \\
-\psi^{\prime} \sin \psi & \psi^{\prime} \cos \psi
\end{array}\right)\left(\begin{array}{cc}
1 & 1 \\
1 & -1
\end{array}\right)\left(I+O\left(x^{-1}\right)\right) h_{0}\left(I+O\left(x^{-1}\right)\right)
$$

and complete the proof of the lemma.

Now, we continue the proof of our main theorem. With the fundamental system of solutions of (2.7) we have found, we can change (2.5) to its corresponding integral form:

$$
\begin{aligned}
& W_{1}(x)=W_{10}(x)+c \int_{\infty}^{x}\left(e^{-\cos \left(2 a^{2} \ln \tau+\phi_{0}\right)}+O\left(\tau^{-1}\right)\right)\left(w_{1}(x) w_{2}(\tau)-w_{2}(x) w_{1}(\tau)\right) \\
& \times\left[\tau^{-3 / 2} g_{1}(\tau) W_{1}+\tau^{-1} g_{2}(\tau) W_{1}^{2}+\tau^{-1} g_{3}(\tau) W_{1}^{3}+\tau^{-2} f_{5}(W)\right] d \tau, \\
& W_{1}^{\prime}(x)=\bar{W}_{10}(x)+c \int_{\infty}^{x}\left(e^{-\cos \left(2 a^{2} \ln \tau+\phi_{0}\right)}+O\left(\tau^{-1}\right)\right)\left(w_{1}^{\prime}(x) w_{2}(\tau)-w_{2}^{\prime}(x) w_{1}(\tau)\right) \\
& \times\left[\tau^{-3 / 2} g_{1}(\tau) W_{1}+\tau^{-1} g_{2}(\tau) W_{1}^{2}+\tau^{-1} g_{3}(\tau) W_{1}^{3}+\tau^{-2} f_{5}(W)\right] d \tau,
\end{aligned}
$$

where

$$
\begin{aligned}
& W_{10}(x)=\int_{\infty}^{x}\left(e^{-\cos \left(2 a^{2} \ln \tau+\phi_{0}\right)}+O\left(\tau^{-1}\right)\right)\left(w_{1}(x) w_{2}(\tau)-w_{2}(x) w_{1}(\tau)\right) g_{0}(\tau) d \tau, \\
& \bar{W}_{10}(x)=\int_{\infty}^{x}\left(e^{-\cos \left(2 a^{2} \ln \tau+\phi_{0}\right)}+O\left(\tau^{-1}\right)\right)\left(w_{1}^{\prime}(x) w_{2}(\tau)-w_{2}^{\prime}(x) w_{1}(\tau)\right) g_{0}(\tau) d \tau .
\end{aligned}
$$


We pay attention to the first equation of (2.19). Noticing that $g_{0}(x)=(2 / 3) a^{3} x^{-1} \cos 3 \phi$ $+O\left(x^{-3 / 2}\right)$ and

$$
\begin{aligned}
w_{1}(x) & \int_{\infty}^{x} \tau^{-1} e^{-\cos \left(2 a^{2} \ln \tau+\phi_{0}\right)} w_{2}(\tau) \cos 3 \phi d \tau \\
= & \frac{1}{4} w_{1}(x) \int_{\infty}^{x} \tau^{-1}\left(\cos \Phi_{2}+\cos \Psi_{2}-\sin \Phi_{2}-\sin \Psi_{2}\right) \\
& \times e^{-(1 / 2)\left(\sin \left(2 a^{2} \ln \tau+\phi_{0}\right)+\cos \left(2 a^{2} \ln \tau+\phi_{0}\right)\right)} d \tau \\
= & \frac{1}{4} w_{1}(x) \int_{\infty}^{x} \frac{\tau^{-1} e^{-(1 / 2)\left(\sin \left(2 a^{2} \ln \tau+\phi_{0}\right)+\cos \left(2 a^{2} \ln \tau+\phi_{0}\right)\right)}}{3-a^{2} \tau^{-1}} d\left(\sin \Phi_{2}+\cos \Phi_{2}\right) \\
& +\frac{1}{4} w_{1}(x) \int_{\infty}^{x} \frac{\tau^{-1} e^{-(1 / 2)\left(\sin \left(2 a^{2} \ln \tau+\phi_{0}\right)+\cos \left(2 a^{2} \ln \tau+\phi_{0}\right)\right)}}{3-5 a^{2} \tau^{-1}} d\left(\sin \Psi_{2}+\cos \Psi_{2}\right) \\
= & O\left(x^{-1}\right),
\end{aligned}
$$

where $\Phi_{2}=3 \tau-a^{2} \ln \tau+4 \phi_{0}$ and $\Psi_{2}=3 \tau-5 a^{2} \ln \tau+2 \phi_{0}$, we can conclude $W_{10}(x)=$ $O\left(x^{-1 / 2}\right)$. We let $V(x)=x^{1 / 2} W_{1}(x)$ and $V_{0}(x)=x^{1 / 2} W_{10}(x)$. Then, the equation corresponding to (2.19) is

$$
\begin{aligned}
V(x)= & V_{0}(x)+c x^{1 / 2} \int_{\infty}^{x}\left(e^{-\cos \left(2 a^{2} \ln \tau+\phi_{0}\right)}+O\left(\tau^{-1}\right)\right)\left(w_{1}(x) w_{2}(\tau)-w_{2}(x) w_{1}(\tau)\right) \\
& \times \tau^{-2}\left[g_{1}(\tau) V+g_{2}(\tau) V^{2}+\tau^{-1 / 2} g_{3}(\tau) V^{3}+f_{5}(W)\right] d \tau \\
= & V_{0}(x)+K(x, V(x)) .
\end{aligned}
$$

To apply the successive approximation method, we define the sequence

$$
V_{n}(x)=V_{0}(x)+K\left(x, V_{n-1}(x)\right), \quad n=1,2, \ldots
$$

Let $q$ be a constant such that

$$
\begin{aligned}
& |c|\left|e^{-\cos \left(2 a^{2} \ln \tau+\phi_{0}\right)}+O\left(\tau^{-1}\right)\right|\left|w_{1}(x) w_{2}(\tau)-w_{2}(x) w_{1}(\tau)\right| \\
& \quad \times\left(\left|g_{1}\right| M_{0}+\left|g_{2}\right| M_{0}^{2}+\tau^{-1 / 2}\left|g_{3}\right| M_{0}^{3}+\left|f_{5}(W)\right|\right) \\
& \leq q \quad \forall x \geq x_{0}, \tau \geq x_{0},
\end{aligned}
$$

and let $M_{0}$ be another constant such that $\left|V_{0}(x)\right|<M_{0}$ for all $x \geq x_{0}$ and $q<M_{0}$. Then,

$$
\left|V_{1}(x)-V_{0}(x)\right| \leq q x^{-1 / 2} .
$$

Assume that

$$
\begin{gathered}
\left|V_{n}(x)-V_{n-1}(x)\right| \leq q x^{-1 / 2}\left(k x^{-1 / 2}\right)^{n-1}, \\
\left|V_{n}(x)\right| \leq M_{0}+q x^{-1 / 2} \frac{1-\left(k x^{-1 / 2}\right)^{n}}{1-k x^{-1 / 2}} \text { for some } n \geq 1,
\end{gathered}
$$


where $k$ is a constant such that

$$
\begin{aligned}
& |c|\left|e^{-\cos \left(2 a^{2} \ln \tau+\phi_{0}\right)}+O\left(\tau^{-1}\right)\right|\left|w_{1}(x) w_{2}(\tau)-w_{2}(x) w_{1}(\tau)\right| \\
& \quad \times\left(\left|g_{1}\right| M_{0}+\left|g_{2}\right| M_{0}^{2}+\tau^{-1 / 2}\left|g_{3}\right| M_{0}^{3}+\left|\frac{d f_{5}}{d W}(W)\right|\right) \leq k, \quad \text { for } x \geq x_{0}, \tau \geq x_{0},
\end{aligned}
$$

then

$$
\begin{gathered}
\left|V_{n+1}(x)-V_{n}(x)\right| \\
\begin{aligned}
\leq-x^{1 / 2} M_{1} \int_{\infty}^{x} \tau^{-2} \mid & g_{1}(\tau)+g_{2}(\tau)\left(V_{n}+V_{n-1}\right)+\tau^{-1 / 2} g_{3}(\tau)\left(V_{n}^{2}+V_{n-1} V_{n}+V_{n-1}^{2}\right) \\
& +\tau^{-1 / 2} \frac{d f_{5}}{d W}\left(W_{n}^{*}\right)|| V_{n}-V_{n-1} \mid d \tau
\end{aligned} \\
\leq-x^{1 / 2} M_{1} \int_{\infty}^{x} \tau^{-2}\left(\left|g_{1}\right|+2\left|g_{2}\right| M_{0}+12 \tau^{-1 / 2}\left|g_{3}\right| M_{0}^{2}+\tau^{-1 / 2}\left|\frac{d f_{5}}{d W}\left(W_{n}^{*}\right)\right|\right) \\
\times q \tau^{-1 / 2}\left(k \tau^{-1 / 2}\right)^{n-1} d \tau \\
\leq q x^{-1 / 2}\left(k x^{-1 / 2}\right)^{n}, \\
\left|V_{n+1}(x)\right| \leq\left|V_{n}(x)\right|+\left|V_{n+1}(x)-V_{n}(x)\right| \\
\leq M_{0}+q x^{-1 / 2} \frac{1-\left(k x^{-1 / 2}\right)^{n}}{1-k x^{-1 / 2}}+q x^{-1 / 2}\left(k x^{-1 / 2}\right)^{n} \\
\leq M_{0}+q x^{-1 / 2} \frac{1-\left(k x^{-1 / 2}\right)^{n+1}}{1-k x^{-1 / 2}} .
\end{gathered}
$$

By the mathematical induction, we have proved that

$$
\left|V_{n}(x)-V_{n-1}(x)\right| \leq q x^{-1 / 2}\left(k x^{-1 / 2}\right)^{n-1} \quad \forall n=1,2,3, \ldots
$$

Therefore, the sequence $\left\{V_{n}(x)\right\}$ uniformly converges and we can conclude that the first equation in (2.19) has a solution satisfying

$$
W_{1}(x)=O\left(x^{-1 / 2}\right)
$$

Similarly, we can apply the same method to the second equation in (2.19) and prove that

$$
W_{1}^{\prime}(x)=O\left(x^{-1 / 2}\right)
$$

Substituting (2.1), (2.4), (2.30), and (2.31) back into transformation (1.3), we obtain the asymptotics stated in Theorem 1.1 and finish the proof.

ACKNOWLeDgment. The authors express their many thanks to Dr. A. R. Its for his comments and encouragement on this work. 


\section{REFERENCES}

[1] A. S. Abdullayev, Justification of asymptotic formulas for the fourth Painlevé equation, Stud. Appl. Math. 99 (1997), no. 3, 255-283.

[2] B. Ganbier, Sur les équations différentielles du second ordre et du premier degré dont l'intégrale générale est à points critique fixés, Acta Math. 33 (1910), 1-55 (French).

[3] W. A. Harris Jr. and D. A. Lutz, A unified theory of asymptotic integration, J. Math. Anal. Appl. 57 (1977), no. 3, 571-586.

[4] S. P. Hastings and J. B. McLeod, A boundary value problem associated with the second Painlevé transcendent and the Korteweg-de Vries equation, Arch. Ration. Mech. Anal. 73 (1980), no. 1, 31-51.

[5] Y. Lu and B. McLeod, Asymptotics of the negative solutions to the general fifth Painleve equation, Appl. Anal. 73 (1999), no. 3-4, 523-541.

[6] B. M. McCoy, J. H. H. Perk, and R. E. Shrock, Time-dependent correlation functions of the transverse Ising chain at the critical magnetic field, Nuclear Phys. B 220 (1983), no. 1, 35-47.

[7] P. Painlevé, Mémoire sur les équations différentielles dont l'intégrale générale est uniforme, Bull. Soc. Math. France 28 (1900), 201-261 (French).

[8] _ Sur les équations différentielles du second ordre et d'ordre supérieur dont lintégrale générale est uniforme, Acta Math. 25 (1902), 1-85 (French).

Youmin Lu: Department of Mathematics and Computer Science, Bloomsburg University, Bloomsburg, PA 17815, USA

E-mail address: y $1 \mathrm{u} @$ bloomu. edu

Zhoude Shao: Department of Mathematics, Millersville University, Millersville, PA 17551, USA

E-mail address: zhoude.shao@mi11ersvi11e.edu 


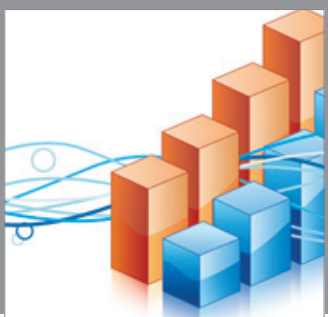

Advances in

Operations Research

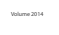

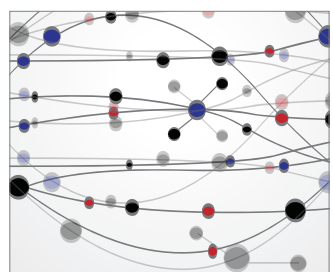

\section{The Scientific} World Journal
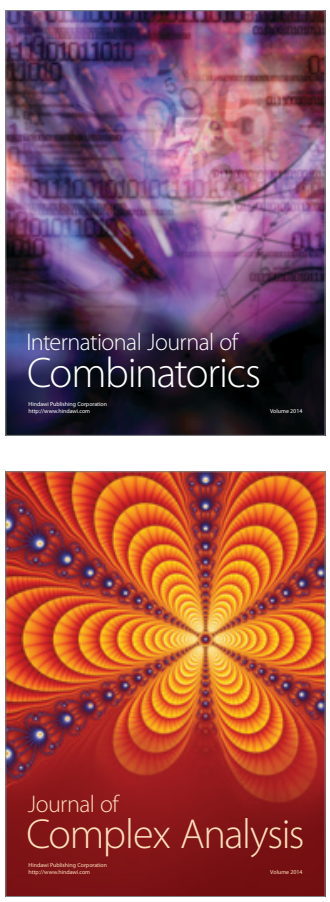

International Journal of

Mathematics and

Mathematical

Sciences
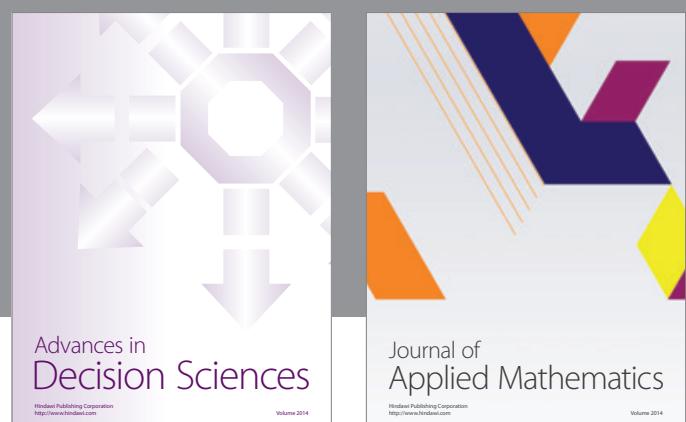

Journal of

Applied Mathematics
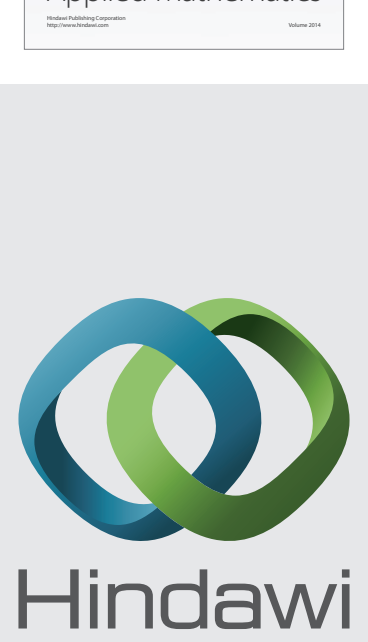

Submit your manuscripts at http://www.hindawi.com
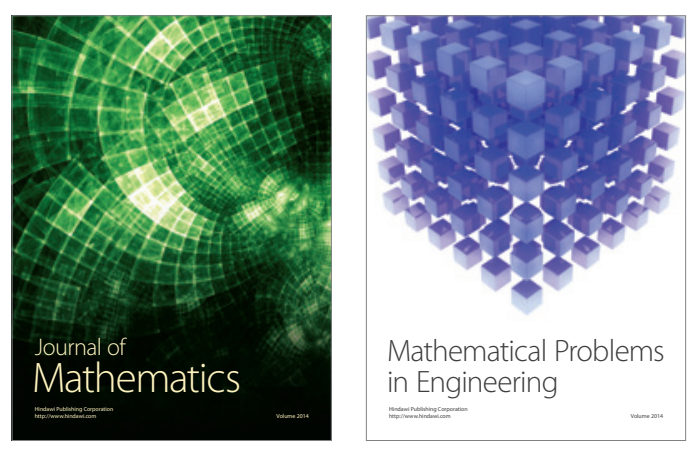

Mathematical Problems in Engineering
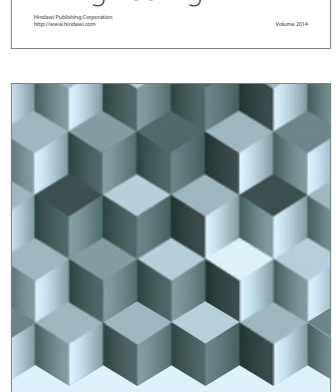

Journal of

Function Spaces
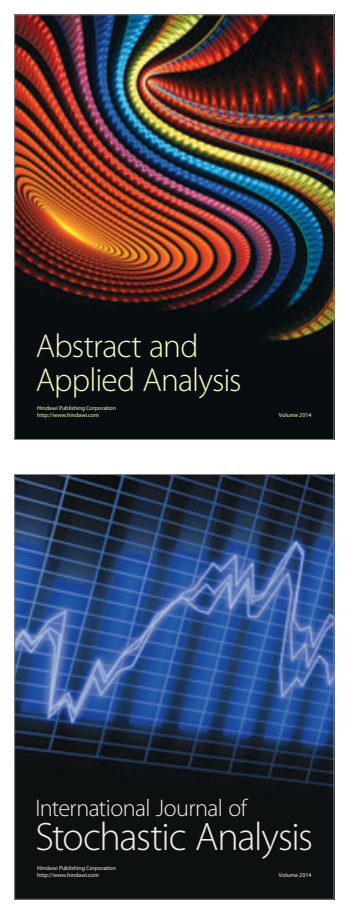

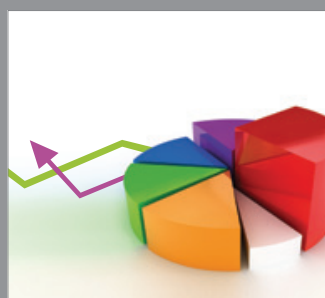

ournal of

Probability and Statistics

Promensencen
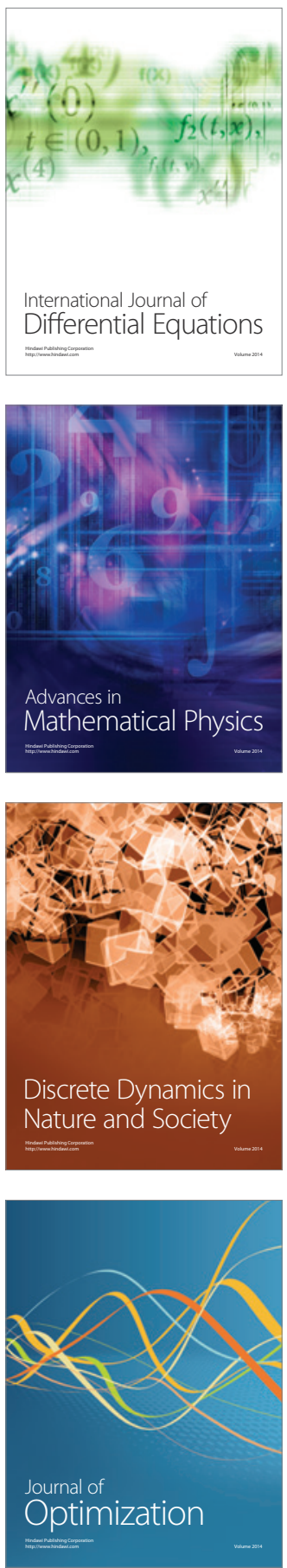